\title{
El enfoque cualitativo en la comprensión de los procesos de cambio en educación.
}

\author{
María Inés Vázquez Clavera ${ }^{1}$
}

\begin{abstract}
Resumen
La mayoría de los países de la región han incorporado el cambio y la mejora como elementos clave de sus agendas educativas. Representan desafíos impostergables para lograr la universalización de la educación básica y la mejora en la calidad de los aprendizajes. Las políticas educativas han priorizado principios como los de inclusión, equidad, diversidad, participación. Uruguay forma parte de este grupo de países. Más allá de los enormes esfuerzos puestos en juego, algunos estudios recientes (INEEd, 2014) ponen en evidencia la distancia existente entre las acciones realizadas y los impactos logrados. Problematizamos aquí el análisis de los procesos de cambio en educación, considerando aspectos como los de cultura organizacional asociados a la construcción de sentido de quienes llevan a la práctica esos cambios.
\end{abstract}

Palabras clave: cambio educativo, cultura organizacional, construcción de sentido. 


\title{
The qualitative approach in understanding the processes of change in education.
}

\begin{abstract}
Most countries in the region have incorporated change and improvement as key elements of their educational agendas. These represent challenges that can't be postponed in order to achieve universal basic education and improvement in the quality of learning. Educational policies have prioritized principles such as inclusion, equity, diversity, participation. Uruguay is part of this group of countries. Beyond the enormous efforts put into this, some recent studies (INEED, 2014) highlight the distance between actions taken and impacts achieved. Here we problematize the analysis of change processes in education, considering aspects such as organizational culture associated with the construction of sense of who put those changes into practice.
\end{abstract}

Keywords: educational change, organizational culture, construction of meaning.

\section{El cambio educativo desde una perspectiva de análisis etnográfico}

Los intentos por registrar una cierta realidad en estudio dependerán del abordaje que utilicemos y del encuadre operacional y epistemológico que definan "las lentes" desde las cuales interpretar contextos, situaciones, fenómenos.

Ruiz Olabuénaga (2012:32) nos recuerda que "un mismo fenómeno cambia de sentido en diferentes situaciones (...) que lo familiar pasa desapercibido hasta que es convertido en extraño (...) que lo esencial de la investigación cualitativa es el análisis objetivo del significado subjetivo".

Es así que nos sentimos interpelados en abordar el estudio y la comprensión de procesos de cambio educativo, desde una mirada que al decir de Nicastro (2006) definimos como contextual y situada. 
Tal como afirma De Souza (2013), ninguna perspectiva que aborde lo social puede tener la pretensión de abarcarla en su totalidad. "La historia humana se constituye de las "constelaciones singulares", de "casos concretos" (...) representan el primer nivel de generalización de conceptos abstractos (...) vinculados a la realidad concreta" (De Souza, 2013:81).

Partiendo de esta advertencia, destacamos que tal vez uno de los principales desafíos del investigador cualitativo sea el no quedar sujeto a lo anecdótico del relato particular, situado, para trascender a un segundo nivel que permita generar diversos diálogos entre: (i) las evidencias, (ii) los referentes teóricos que son en definitiva los que permiten recuperar una visión global y (iii) los procesos metodológicos como aportes instrumentales que permitan ir armando una cierta trama argumental a partir del análisis.

La perspectiva de trabajo que elegimos en este caso para profundizar en la comprensión de los procesos de cambio en educación, nos enfrenta a la necesidad de precisar conceptos como el de cultura organizacional, para luego acceder a niveles más específicos de análisis, asociados con la "construcción de sentido" o sensemaking ( Weick, 1995).

"Lo concreto aparece como un punto de llegada y como un punto de partida, mediado por teorías y métodos que conducen a la comprensión. (...) lo real, como un dato inmediato, reaparece mediatizado por la teoría" (De Souza, 2013:95).

Diferentes autores que han abordado el estudio de los procesos de cambio educativo (House, 1981; Bourdiau, 1985; Butelman, 1996; Perrenoud, 1998, Morin, 1999; Guarro, 2005 entre otros), ponen en evidencia las diversas perspectivas desde las cuales son analizadas estas dinámicas, así como las múltiples interpretación que pueden surgir de los estudios que abordan un mismo fenómeno.

Según Schutz (1964; citado por De Souza, 2013), se pueden identificar una serie de factores que afectan las "tipificaciones" que las personas realizan todo el tiempo sobre aspectos cotidianos. Entre otros aspectos el autor destaca el peso de sus experiencias biográficas, el stock de conocimiento con el que interpretan las distintas situaciones, la capacidad empática y reflexiva de cada actor social, el tipo de vínculo que se logra establecer con los diferentes colectivos de pertenencia. La lista de factores es extensa y abarcable según la amplitud de la mirada con la que se trabaje en cada caso.

En esta línea Fullan (2002) distingue entre lo que denomina "cambio subjetivo", asociado a la representación que tanto las personas como las instituciones tienen sobre los procesos de cambio en los que participan y el "cambio objetivo" relacionado con profundos procesos de transformación de la matriz cultural o "reculturación", que llevan a la institucionalización de lo novedoso. 
Adherimos a esta distinción, entendiendo que la consolidación de los procesos de cambio requieren de verdaderas transformaciones en la forma de percibir y entender las prácticas cotidianas. Es así que destacamos momentos o fases del cambio en cuyo desarrollo se facilita (o dificulta) el fenómeno de la reculturación.

Todo proceso de cambio genera esfuerzos, aprendizajes y también resistencias que activan diversas fuerzas - de apoyo unas y de rechazo otras - definiendo así procesos de avance y retroceso íntimamente asociados a cada contexto (Vázquez, 2009). Los escenarios educativos no son ajenos a estas dinámicas.

\section{Transformaciones en la matriz cultural}

Retomando la perspectiva que propone Fullan (2002), destacamos la importancia de articular desde el análisis aspectos visibles de los procesos de cambio con otros no tan evidentes, asociados a las formas de entender y significar los cambios en quienes tendrán a su cargo su puesta en marcha y su consolidación.

Según Fernández (2007:104) "matriz cultural" refiere a cercos que "configuran los sistemas de prioridades de las personas, organizan sus prácticas sociales y sus prácticas de sí (...) lo valorado y lo devaluado (...) los usos sociales".

Al mirar las políticas del cambio desde la imagen del "iceberg" (Vandenberghe y Staessens, 1991), la región se ha caracterizado por reiterados ciclos de inicios y finalizaciones de procesos de cambio dentro de una misma década, sin que hayan quedado en evidencia los logros educativos alcanzados en cada caso. Estos ciclos parecen estar más relacionados con el traspaso de autoridades de un período al siguiente que con la consolidación de fases del cambio anunciado o el inicio de otros a partir de su institucionalización.

Los proyectos de cambio se presentan al análisis como piezas intercambiables de un puzle organizado por políticas educativas cíclicas que parecen no compartir una visión global y prospectiva de lo educativo. La sucesión de este tipo de situaciones es lo que Bauman (2005) asocia con la fragilidad de las identidades colectivas que finalmente no logran sostener en el tiempo este constante "comenzar de nuevo".

Asistimos así a una cierta banalización del cambio en educación, naturalizándose lógicas como las que advierten que los cambios prometidos finalmente nunca se producen (al menos no en los cimientos del iceberg).

Esta operativa en las políticas de cambio que podríamos definir como superficial y cíclica, impide el desarrollo y consolidación de verdaderos procesos de reculturación, lo que estaría evidenciando que estamos ante propuestas que se vuelven más cosméticas que reales y en tal caso, altamente vulnerables a permanecer en el tiempo. 
Tampoco se identifican estrategias, dispositivos o políticas educativas que retomen esta dificultad de llevar los procesos de cambio a la práctica, procurando alternativas para su superación. La ausencia de registros sistemáticos de las innovaciones puestas en marcha favorecen esta indefinición de disfuncionalidades y la concreción de propuestas que permitan su superación (Vázquez y Borgia, 2014).

Bauman (2012:45) analizando los escenarios educativos nos advierte, " una cantidad cada vez más grande de información se distribuye a una velocidad cada vez más alta(...)esto tiene consecuencias en el modo en que nos relacionamos con el conocimiento, con el trabajo y con el estilo de vida en un sentido amplio".

Surgen así múltiples propuestas de cambio y mejora que no logran consolidarse y conseguir los impactos previstos al no haber logrado incidir en la matriz cultural de los escenarios educativos en cuestión.

\section{Las dinámicas de construcción de sentido como anclaje al cambio}

Según Weick (1995), la construcción de sentido refiere a la forma en que las personas interpretan lo que son y lo que hacen, aspectos que condicionan fuertemente la actitud y disposición que cada uno adopte en su escenario laboral.

El sentido es "aquella idea hacia la cual nos dirigimos y en relación a la cual juzgamos y decidimos. (...) sirve como marco interpretativo, estable y global para cualquier acción o pensamiento , porque da forma" (Aizpún, 2009:151).

Desde esta perspectiva, la construcción de sentido aporta al proceso de entendimiento de la propia tarea y la de otros en un espacio social concreto, lo que aporta al fenómeno de identidad institucional. Son procesos que no solo influyen en la imagen que se tenga de sí mismo sino que también influyen en los tipos de vínculos que se logran establecen con la tarea y con los demás miembros de la institución.

Este conjunto de fuerzas y dinámicas imperceptibles a simple vista, representan el entramado que va conformando y definiendo la matriz cultural de ese espacio educativo. Desde esta perspectiva es que Walsh \& Ungson (1991) citado por Weick (1995:38) ${ }^{2}$ definen a la organización, como una red de intersubjetividades que comparten sentido a través del lenguaje común y las interacciones.

Todo cambio requiere de "actores educativos", teniendo en cuenta tal como lo define Aizpún (2009), que el actuar significa "moverse con sentido", en un marco contextual específico, marcando una cierta direccionalidad. Este atributo de conexión entre las personas y las acciones que realizan adquiere especial 
relevancia en aquellos escenarios en procesos de cambio, en los que las personas se encuentran implicadas.

Solo a partir del trabajo colaborativo, sustentado desde el sistema con tiempos y espacios viables para su consolidación, es posible acceder a verdaderas redes que habiliten la creación, difusión y transmisión de conocimiento (Casas, 2001).

Resulta interesante destacar que las dinámicas educativas que tradicionalmente han sido explicadas desde la incidencia de aspectos formales y normativos (propuestas curriculares, organigramas, reglamentos, etc.), comienzan a ser analizadas desde las configuraciones de interacción que se producen en los colectivos, así como las estructuras cognitivas que se generan entre ellos. Autores tales como Castoradis (1983) o Bourdieu (1991) refieren al "imaginario social" o al "habitus", haciendo alusión a sistemas estructurantes de significados colectivos que son los que condicionan las prácticas de esos espacios compartidos.

Se considera imposible otorgar sentido a aquellas propuestas o acciones digitadas "desde fuera", con las que no se logra una clara identificación. "Si no andamos en una dirección no llegamos a ningún sitio, no hay acción sin sentido" (Aizpún, 2009:222).

\section{Criterios metodológicos para el análisis cultural de los procesos de cambio}

"La promoción de pensamiento crítico representa un camino que permite la articulación entre pensamiento y acción. Para lograrlo, es necesario trabajar desde las propias representaciones, tomándolas como principal insumo desde el cual generar acciones de reflexión colectiva que permitan revisar los supuestos de los que se parte y analizar alternativas que habiliten la consolidación de nuevos enfoques" (Vázquez, 2014:10).

Para lograrlo y como paso previo, es necesario hacer visible aspectos de las dinámicas colectivas que por su característica abstracta, no siempre se hacen evidentes en un primer acercamiento de análisis. No se puede actuar sobre aquellos aspectos de la realidad que no son percibidos como presentes.

Una de las vías de acceso a estos procesos colectivos se logra a partir de entrevistas en profundidad aplicadas a informantes clave identificados en cada contexto. El estudio de estas evidencias se logran a través del análisis del discurso (Van Dijk, 2008), entendiéndolo como "un suceso de la comunicación" a partir del cual las personas comparten ideas, creencias, expectativas, en el marco de situaciones sociales específicas.

Como estrategia metodológica para organizar el proceso de análisis, hemos adoptado el modelo propuesto por Spradley (1980), el cual permite profundizar 
en la identificación de dominios, taxonomías y componentes que integran y le dan sentido a un cierto argumento (discurso), que orienta tanto las prácticas individuales como otras colectivas.

Los dominios representan una categoría general de significados que engloba a otras menores que surgen a partir del análisis de los discursos. Los términos inclusivos relacionan a los subcomponentes de cada dominio con atributos que surgen desde el análisis. Finalmente las relaciones semánticas aportan la connotación que adquieren esos vínculos para la persona consultada, otorgándole un peso "positivo" o "negativo", que seguramente afectará su disposición (afín o refractaria) ante el tema en cuestión.

Este proceso de análisis brinda aportes sustanciales que permiten comprender no solamente el "sentir" sino también la "predisposición ante el actuar" de los actores educativos implicados en escenarios de cambio. Entendemos fundamental conocer y comprender la postura que cada persona manifiesta sobre los procesos organizativos en los que se encuentra implicada, como primer paso para estimar los niveles de impacto previstos en cada contexto.

El análisis desde esta perspectiva, permite configurar "diagramas de árbol" conteniendo los argumentos que sustentan las prácticas (individuales y/o colectivas), identificando aspectos de la propia matriz cultural de una cierta institución en estudio (aspectos valorados/devaluados, prioridades, supuestos de base, etc.).

Al tratarse de un modelo que toma como principal insumo la palabra de los implicados, representa una puerta de acceso directa al análisis de creencias, supuesto y representaciones que consideramos componentes sustanciales para comprender las lógicas que sustentan las prácticas colectivas asociadas a los procesos de cambio.

Como decíamos antes, la visión contextual y situada que aportan estos abordajes de análisis, permiten hacer visible y evidente aspectos que, desde otros enfoques más generales podrían pasar totalmente desapercibidos.

Hemos tenido la oportunidad de aplicar esta modalidad de análisis en diversos estudios asociados con procesos de mejora y cambio en su relación con aspectos de la cultura (Vázquez, 2009; Vázquez y otros 2013; Vázquez y Rodríguez, 2014). Todos ellos nos han permitido validar la pertinencia de este camino metodológico en la comprensión de procesos de cambio en escenarios educativos.

Este proceso analítico que visualizamos como espiralado, nos ha habilitado a ir y volver de lo particular a lo más general, evitando así quedar atrapados en lo particular y anecdótico de situaciones concretas (Vázquez, 2013). 
El análisis contextual y situaciones de diferentes fenómenos sociales, habilita a la comprensión del fenómeno en estudio como paso previo y fundamental para luego promover acciones que permitan su revisión y mejora.

\section{Conclusiones}

En términos generales podríamos jerarquizar cuatro aspectos relevantes como aportes de este trabajo. Ellos son:

- Todo proceso de cambio en educación incluye aspectos estructurales, evidentes al análisis (propuesta curricular, organigramas, normativas, perfiles, etc.) y otros procedimentales no tan obvios desde las perspectivas globales de las políticas educativas .

- Los escenarios educativos que oficina como plataformas del cambio, no son "neutros", sino que condicionan - facilitando o dificultando- los procesos que se intentan implementar desde el sistema. Representan las unidades operativas desde las cuales se pone en práctica el cambio y en tal caso, entendemos como estratégico lograr un mejor conocimiento de sus dinámicas internas, su back stage.

- Cada agente educativo (docente, directivo, estudiante, etc.) le otorga un cierto sentido a la propuesta de cambio lo que termina afectando los niveles de involucramiento y de apoyo brindados. Estos procesos de construcción de sentido son los que definen aspectos de la cultura organizacional, así como las dinámicas colectivas logradas a partir de ellos.

- Los enfoques del análisis contextualizado en las lógicas específicas de los actores educativos que participan del cambio permiten alcanzar un doble propósito: (i) superar ciertas relaciones de inherencia propias de otros enfoques macroestructurales y (ii) alcanzar una mayor comprensión de procesos que tienden a pasar desapercibidos por su escasa visibilidad.

- El análisis de la construcción de sentido basado en el modelo de Spradley, es considerado por nosotros como una alternativa metodológica desde la cual lograr una mayor comprensión de factores contextuales asociados a los procesos de cambio. Representa una perspectiva de análisis que entendemos valiosas para avanzar en la consolidación de políticas educativas asociadas al cambio. 


\section{Revista de}

\section{Referencias bibliográficas}

Aizpún, T. 2009. Gramática de la vida. La ética como creatividad. Madrid: Endymion

Baumn, Z. 2005. Identidad. Madrid: Losada

Bauman, Z. 2012. Sobre la Educación en un mundo líquido. Barcelona: Paidos

Bourdieu.1991. El sentido práctico. Madrid: Taurus

Casas, R. 2001. La formación de redes de conocimiento >>. Universidad Autónoma de México: Anthropos

Castoradis. 1983. Las instituciones imaginarias de la sociedad. Barcelona: Tusquets

De Souza, M. 2013. La artesanía de la investigación cualitativa. Bs. As. : Lugar Editorial

Fernández, A. 2007. Las lógicas colectivas. Imaginarios, cuerpos y multiplicidades. Bs. As.: Biblos

Fullan, M. 2002. Los nuevos significados del cambio en educación. Barcelona:Octaedro

INEEd. 2014. Informe sobre el estado de la educación en Uruguay. Montevideo: INEEd

Nicastro, S. 2006. Revisitar la mirada sobre la escuela. Santa Fé: Homo Sapiens

Ruiz, J. 2012. Metodología de la investigación cualitativa. Bilbao: Universidad de Deusto

Spradley. 1980. Citado por Rodriguez Gómez y otros. (1999) «Metodología de investigación cualitativa. Málaga: Aljibe

Vandenberghe, R. \& Staessens, K. 1991. Vision as a core component in school culture. Chicago: IL

Van Dijk, T. 2008. El discurso como estructura y proceso. Barcelona: Gedisa

Vázquez, M. 2009. La promoción del cambio organizacional a partir de la autoevaluación. Tesis doctoral. Barcelona: Universidad Autónoma de Barcelona

Vázquez, M. 2013. Propuesta metodológica para analizar aspectos de la cultura asociados al Desarrollo Organizacional. Congreso Internacional EDO. Barcelona: Universidad Autónoma de Barcelona.

Vázquez, M. et al. 2013. Competencias del equipo directivo: identificando factores organizacionales asociados al fenómeno de la inclusión educativa. En: VI Congreso Internacional sobre Dirección de Centros Educativos. San Sebastián: Universidad de Deusto 


\section{María Inés Vázquez Clavera}

El enfoque cualitativo en la comprensión de los procesos de cambio en educación. Artículo producto de la investigación.

Vázquez, M. 2014. Propuesta metodológica para analizar aspectos de la cultura asociados al desarrollo organizacional. Ponencia. Congreso Internacional EDO. Barcelona: UAB

Vázquez, M \& Borgia, F. 2014. Educación en Uruguay: principales ejes actuales de discusión. Observatorio Uruguay de Politicas Pùblicas. Montevideo: AGEV-OPP. Disponible en: http://agev.opp.gub.uy/observatorio_docs/publico/discusion_ politicas_educacion_uruguay_2014.pdf Fecha de acceso: 10/10/15

Vázquez, M \& Rodríguez, E. 2014. Estudio de las prácticas pedagógicas y las habilidades profesionales docentes en el marco de las políticas de inclusión educativa en Uruguay. Informe de investigación. Montevideo: Universidad ORT Uruguay

Weick, K. 1995. Sensemaking in Organizations. London: SAGE Publications Ltd. 